\title{
Imaging Mass Spectrometry: Hype or Hope?
}

\author{
Ron M. A. Heeren, Donald F. Smith, Jonathan Stauber, \\ Basak Kükrer-Kaletas, and Luke MacAleese \\ FOM Institute for Atomic and Molecular Physics, Amsterdam, The Netherlands
}

\begin{abstract}
Imaging mass spectrometry is currently receiving a significant amount of attention in the mass spectrometric community. It offers the potential of direct examination of biomolecular patterns from cells and tissue. This makes it a seemingly ideal tool for biomedical diagnostics and molecular histology. It is able to generate beautiful molecular images from a large variety of surfaces, ranging from cancer tissue sections to polished cross sections from old-master paintings. What are the parameters that define and control the implications, challenges, opportunities, and (im)possibilities associated with the application of imaging MS to biomedical tissue studies. Is this just another technological hype or does it really offer the hope to gain new insights in molecular processes in living tissue? In this critical insight this question is addressed through the discussion of a number of aspects of MS imaging technology and sample preparation that strongly determine the outcome of imaging MS experiments. (J Am Soc Mass Spectrom 2009, 20, 1006-1014) (c 2009 Published by Elsevier Inc. on behalf of American Society for Mass Spectrometry
\end{abstract}

$\mathrm{I}$ a picture speaks a thousand words then an imaging mass spectrometry experiment is a book with many chapters. Each page of the book contains different descriptive pictures of an independent molecular distribution on a complex surface such as a histopathological tissue section. In this way new molecular anatomical atlases can be created. This opens the door for molecular histology in biomedicine. Historically there are many different applications of imaging MS [1]. In the field of semiconductor production and research it has been one of the tools of choice for surface contamination analysis. It has been used in the study of aging of old-master paintings, polymeric surfaces, geological samples, and forensic research. The biggest driver for the recent rapid developments is the prospect of the application of imaging MS directly on biological tissue sections [2-6] and single cells and the full integration within the proteomics field. For that reason the discussion in this presentation will be restricted to the implications, challenges, opportunities, and (im)possibilities associated with the application of imaging MS of biomedical tissue.

The advantages of using a mass spectrometer for molecular imaging in the discovery phase of any biomedical experiment are wide-ranging in scope. It eliminates the need for labeling because the molecular mass is used as an endogenous label. This leaves the biomolecules of interest functionally unmodified. In this way it removes the interference of potential fluorescent labels

Address reprint requests to Prof. Ron M. A. Heeren, FOM Institute for Atomic and Molecular Physics, Macromolecular Ion Physics, Sciencepark 113, 1098 SX Amsterdam, The Netherlands. E-mail: heeren@amolf.nl with the biological function. Imaging mass spectrometry also allows the detection of post-translational modifications (PTMs) given that these generally involve mass changes. Often it is not possible to generate antibodies that allow the (labeled) visualization of the PTM distribution directly in tissue. With a mass spectrometer it is "easy" to see the location of the mass change. An additional advantage is that mass spectrometry provides multiplexed information from a surface since for each peak in the mass spectrum an image can be generated. Many commercial instruments now offer imaging capabilities and, as a result, the field is evolving rapidly and many new groups are now getting involved with this exciting technology.

Imaging MS is a booming field, as illustrated by the rapidly increasing number of publications as sketched in Figure 1. The potential of the technology is clear: it offers a new tool to trace various compounds and metabolites. Yet as with many new technologies there is a risk that expectations are raised that will be difficult to meet because of several technological obstacles. That raises the question: hype or hope?

The hope of biomedical imaging mass spectrometry can be summarized in a single sentence: It is the hope that we will be able to cure neurodegenerative diseases, cancer, and aggressive viral infections through better fundamental understanding of the morphology and molecular pathology of the diseases. Imaging mass spectrometry has the power to provide that molecular insight. In that light it can be considered a discovery tool. It is claimed to be capable of discovering new molecular distributions that when compared to existing immunohistochemical images can indicate which other molecules are involved in a (c) 2009 Published by Elsevier Inc. on behalf of American Society for Mass Spectrometry. $1044-0305 / 09 / \$ 32.00$

doi:10.1016/j.jasms.2009.01.011
Published online March 21, 2009 Received October 10, 2008 Revised January 16, 2009 Accepted January 16, 2009 


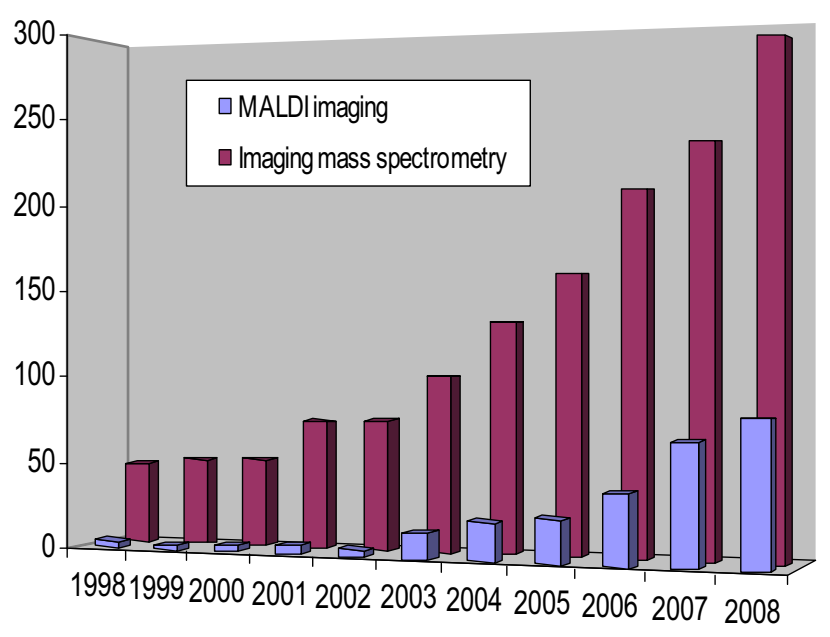

Figure 1. Development of imaging mass spectrometry publication numbers since 1998. Results based on an ISI keyword search using imaging mass spectrometry and MALDI imaging.

certain disease pathology or drug metabolism. The question arises: "Is it there yet?"

\section{Application Areas}

Historically, imaging mass spectrometry is applied in material sciences where Secondary Ion Mass Spectrometry (SIMS) is used to examine the distribution of inorganic species on surfaces. SIMS is also used to examine biological surfaces, but never made a major impact in biological research [7]. LAMMA was one of the earlier laser-based imaging MS technologies, but its application remained restricted to material science, small molecules, and-again-inorganic species. Applications for relevant biomedical studies were not possible until the conception of desorption and ionization technologies such as matrix-assisted laser desorption/ ionization (MALDI) that enabled the analysis of intact biomolecular ions. Approximately 10 years after the first MALDI paper [8] the field of biomolecular imaging mass spectrometry started to emerge [2]. With new sample-handling technologies, innovations in mass spectrometers in this new field influenced other imaging MS applications, as can be seen in Figure 1. To address the question "hype or hope?" it is imperative to explore what the most strongly growing application areas for imaging mass spectrometry are. Three biomedical application areas fit that criterion: proteomics, lipidomics, and pharmacology. The illustration in Figure 2 shows three images from these application areas. Imaging MS in proteomics is often advertised for the visualization of peptide and protein biomarker patterns specific to disease. Comparative image analysis of healthy and diseased tissue sections targets the visualization of aberrant molecular pathology. Imaging in lipidomics can be used to visualize intact lipid molecular distributions and determine head group identity, acyl chain lengths, and degrees of unsaturation on single-cell surfaces. Pharmaceutical research uses imaging MS complementary to whole body autoradiography in both research and the developmental phase of new drugs [9]. MS imaging is particularly interesting because it has the potential to image simultaneously the drugs and their metabolites. MALDI MS imaging offers the possibility not only of examining and tracking drug metabolism in whole animal sections, but also of following polymer substrate degradation and drug release.

Proteomics, lipidomics, and pharmacokinetics incite the biggest interest in the life science community because combined, they can be used to assess the effects of drugs on proteome and lipidome. This sketches a powerful potential and raises expectations of the technology. The question is whether the technology is already mature enough to fulfill this potential. A number of critical elements exist in any imaging MS (IMS) workflow that can strongly affect the outcome of an experiment. In this critical insight we will describe a number of issues that are currently addressed and discussed within the imaging MS community. Sample preparation, ion suppression, sensitivity, and data analysis are aspects of imaging mass spectrometry that merit critical evaluation. Together they strongly influence the outcome and relevance of an imaging experiment. New technologies are continuously added to the imaging MS toolbox to address these issues. There is a strong need to combine these novel imaging tools with existing molecular imaging techniques such as MRI and immunohistochemistry to make IMS an accepted molecular pathology technique in biomedical research.
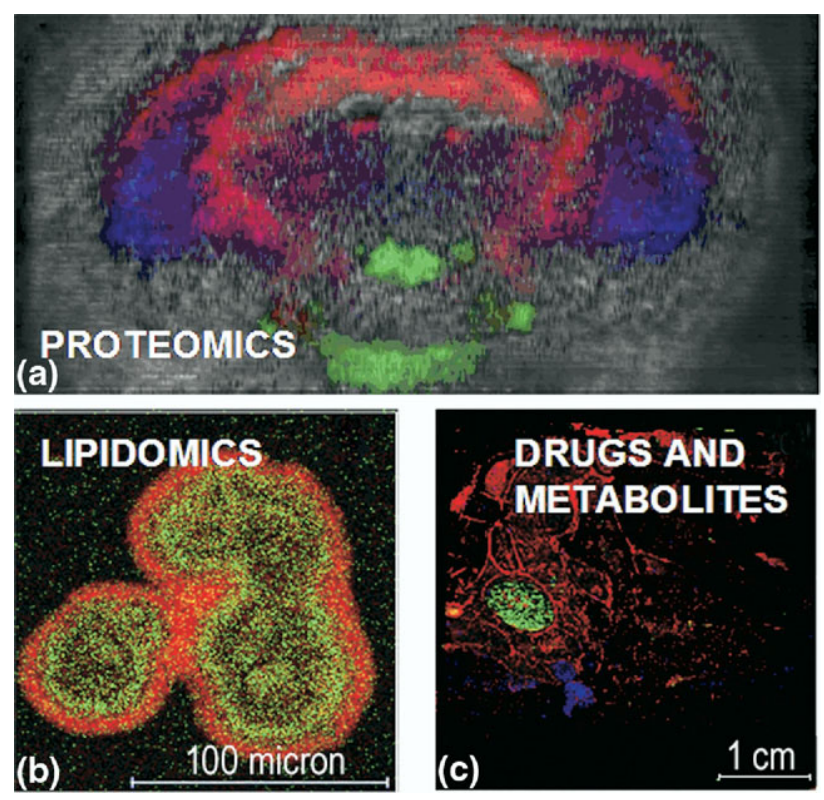

Figure 2. Main biomedical aapplication areas of imaging mass spectrometry, proteomics, lipidomics, and drugs and metabolite imaging. (a) The distribution of a number of endogenous neuropeptides on a rat brain section. (b) The distribution of lipids of the surface of single neuroblastoma cells. (c) The distribution of a metabolite in the kidney of a whole body cross section of a rat. 


\section{State-of-the-Art MS Imaging Technology}

Technological developments have long driven imaging MS. Relatively recently a set of new tools have been added to the MS imaging toolbox. MALDI-time-offlight (ToF) MS-based imaging has become a routine tool from the technological perspective. Its application is limited only by sample quality and reproducible preparation protocols that we will discuss later. In this section three new instrumentation developments are discussed that can be seen as breakthrough technologies in IMS defining the state of the art. Each of them is aimed at improving the data quality and information content resulting from an imaging MS experiment. The first development is the microscope mode approach to improve speed and spatial resolution of an IMS measurement. The second instrumental platform that will further imaging MS is Fourier-transform ion cyclotron resonance (FT-ICR)-MS that addresses the lack of mass spectral resolution and mass accuracy combined with tandem MS (MS/MS) identification possibilities. The last emerging technology is the use of ion mobility separation in IMS experiments that introduces gasphase separation capabilities to distinguish isobaric compounds based on their different molecular conformations. Each of these new developments has clear potential to increase the information content of imaging MS experiments. They will also result in even larger datasets than those obtained with current MALDI-ToF imaging. In the subsections below we will discuss these three new developments individually.

\section{Spatial Resolution: Microscope Mode Imaging MS}

Spatial resolution has long been a limiting factor. Conventional approaches in MALDI-ToF imaging are based on the microprobe approach. There a laser or ion beam is focused to a small spot from which a single mass spectrum is acquired. An image is generated by rastering this spot in a predefined manner over the surface, probing the surface and collecting spectra at different locations. The spatial resolution is determined by the size of the probe beam. In commercial instruments the physical size of the laser spot is limited to around 10 $\mu \mathrm{m}$. On commercial instruments most experiments are performed with a laser spot size between 50 and 250 $\mu \mathrm{m}$. This choice is determined by sensitivity and the time required to complete an experiment. Special confocal type objectives [10] can reduce the spot size to 1 $\mu \mathrm{m}$, although there is substantial debate as to whether the laser threshold fluence required for the use of these small spots with MALDI is not too high for intact molecular analysis directly from tissue. Initial experiments have shown the capability to acquire highresolution images of smaller species. An alternate approach to increase spatial resolution using conventional MALDI-ToF instruments is the oversampling approach introduced by the Sweedler group [11]. In their ap- proach the incremental movement of the laser probe spot is smaller than the spot diameter. After all sample has been consumed at the first spot each incremental step delivers information from a much smaller area. As a result the spatial resolution can be increased. The limited MS/MS possibilities and the total sample consumption are two drawbacks of this approach.

The achievable spatial resolution in imaging MS can also be increased through the use of microscope mode imaging mass spectrometry [12]. This method uses high-quality ion optics that provide an astigmatic projection of ions generated at the surface on the detector of the ToF-MS. This implies that the spatial distribution of the ions stays intact while they are flying through the mass spectrometer, resulting in traveling molecular images. A detector that can simultaneously acquire arrival time and arrival position $\left(\mathrm{x}, \mathrm{y}\right.$, and $\left.\mathrm{t}_{\mathrm{TOF}}\right)$ of the ions at the end of the flight tube can directly acquire these molecular images, essentially taking molecular snapshots. The spatial resolution is defined by the quality of the ion optics, the ion optical magnification, and the pixel size on the detector. The implementation described in Luxembourg et al. [12] delivers pixel sizes that are $600 \mathrm{~nm}$ by diameter. An added advantage is that a larger field of view in acquired simultaneously as a result of direct multiplexed detection of many pixels at once. This dramatically increases the throughput of high spatial resolution imaging MS. A 200- $\mu$ m diameter area can be analyzed with a 600-nm pixel size in $1 \mathrm{~s}$ without fully depleting the surface. This implies that the surface can be reanalyzed with an additional technique, either optically or with another imaging MS step. As an example: Figure 2a was measured in the microscope mode in $2.5 \mathrm{~h}$ and covered an area of $1.5 \times 2.5 \mathrm{~cm}$ with $600-\mathrm{nm}$ pixels and a resolving power (determined by the number of pixels required to resolve a feature) of 2-4 $\mu \mathrm{m}$. The total dataset size was 200 Gbyte. This approach has one other advantage. It also allows the use of desorption and ionization beams that are difficult to focus to submicron spot sizes for high spatial resolution analysis. An example is the use of IR-MALDI imaging, with resolutions below the diffraction limit of the optical system used. It allows IR imaging with identical spatial resolution as UV-based MALDI imaging, even though the wavelength is an order of magnitude longer [13].

Irrespective of which method is used to increase the spatial resolution, sensitivity and spatial resolution remain intimately coupled. Only few molecules are available for desorption and ionization if the spatial resolution would increase to $10 \mathrm{~nm}$ or below. This implies a need for single-ion detection MS imaging. It is obvious that we are not there yet, although new developments in detection technology are moving this field forward. The other limiting factor for high spatial resolution analysis concerns the enormous dataset sizes generated when large areas need to be imaged. Imaging a whole rat section $(\sim 16 \times 4-\mathrm{cm}$ area $)$ with $100-\mathrm{nm}$ pixels would result $6.4 \times 10^{11}$ spectra. This would result in a single 
dataset that requires its own storage system, even when the spectra are reduced to 1 kbyte. This is clearly not achievable with current technology.

\section{Spectral Resolution: FT-ICR MS Imaging}

High-quality images alone are not enough. Compound identification accuracy is equally as important as-if not more important than-spatial resolution. MS imaging methods such as TOF-SIMS and microscope mode MALDI-TOF offer high spatial resolution but lack the mass resolving power and mass accuracy needed for the identification of all observed peaks. MALDI-TOF MS provides a good compromise between spatial resolution and mass resolving power; however, $\mathrm{MS}^{n}$ experiments can be difficult. FT-ICR MS allows spatial resolution similar to that of conventional microprobe MALDI-TOF, with the high mass accuracy and ultrahigh mass resolving power necessary for unequivocal identification of measured masses and use of different $\mathrm{MS}^{n}$ techniques (e.g., sustained off-resonance irradiation collision-induced dissociation [SORI-CID], infrared multiphoton dissociation [IRMPD], and electron capture dissociation [ECD]) [14]. Figure 3 demonstrates the need for ultra-high mass resolving power for identification of species on a rat brain tissue [15]. Insets a and $\mathrm{d}$ in Figure 3 show a selected region from summed spectra for two halves of a rat brain; inset a was analyzed by FT-ICR MS and inset $d$ by TOF-MS. FT-ICR identifies multiple peaks over the mass range, whereas the TOF-MS cannot resolve even the nominal masses. Moreover, inset $\mathrm{b}$ shows that the three species resolved at $\mathrm{m} / \mathrm{z}$ 1293 show unique spatial distributions in the rat brain and that the lower mass resolving power of TOF-MS (see inset e) yields a combined spatial distribution.

The power of FT-ICR MS to image isobaric species on tissue has been demonstrated [15-19]. In addition, each of these studies used MS/MS to positively identify peaks from the mass spectrum. Although FT-ICR MS offers the benefits of high mass accuracy, ultra-high mass resolving power, and multiple MS/MS techniques, it also has inherent disadvantages. Time of analysis becomes an issue for FT-ICR MS at very high mass resolving powers, especially if the experiment is done serially. Interlaced experiments-that is, external accumulation during the excite/detect events- help to shorten experiment times. Similarly, data size is also of concern at very high mass resolving powers, even more so than for typical TOF-MS imaging experiments. Finally, the singly charged ions predominantly produced by MALDI hamper ECD MS/MS fragmentation and also limit the mass range for FT-ICR MS. The analytical capabilities for FT-ICR MS would be greatly enhanced when moving to stronger magnetic fields. Imaging mass spectrometry in particular would benefit with either enhanced resolution (at the expense of throughput) and speed of analysis (at the expense of mass resolution) because the transients become shorter at the same resolution as the ion cyclotron frequencies increase with magnetic field.

\section{Conformational Resolution: Ion Mobility in MS Imaging}

The combination of ion mobility $[20,21]$ in a MALDI-TOF instrument is one of the breakthrough technologies for mass spectrometry imaging and its application. Ion mobility mass spectrometry (IM-MS) is described as a twodimensional gas-phase separation technique using the $\mathrm{m} / \mathrm{z}$ ratio and conformational separation in a drift cell. The ion mobility drift cell region is an electric field and gas-filled region that separates molecules such as nucleotides, lipids, peptides, and proteins by their velocity based on their collision cross section. Inside the drift cell region, ions acquire an average drift velocity based on their collision cross section to charge ratio, thus allowing the separation of molecules according to their conformation. Since the commercialization of different ion mobility cells, a large number of applications have been described in the study of complex mixtures. The two-dimensional separation allows the separation of isobaric compounds, offering new interesting applications. MALDI ion mobility mass spectrometry allows classically top-down protein sequencing and structural determination of noncovalent complexes. When combined with imaging mass spectrometry it offers the possibility of directly indentifying protein conformers, as well as isobaric and low-abundance species in complex mixtures such as tissue sections. As such, IM-MS can be directly used for biological and clinical proteomics studies by MALDI imaging analysis. Because of the separation of isobaric lipids and peptides, a larger number of molecules have been detected and identified directly on-tissue sample $[22,23]$. The use of ion mobility is suitable for the direct profiling of tissue, in lipidomics and proteomic studies.

The ion mobility MSI is suitable for other studies corresponding to the detection and imaging of large proteins. Large proteins $(>80 \mathrm{kDa})$ are usually difficult to detect in classical MALDI experiments. The use of enzymes like trypsin to digest intact proteins offers new perspectives in the direct on-tissue identification of these proteins, in frozen as well as formalin-fixed paraffin-embedded (FFPE) tissue analysis. The consequence of digestion is the increase the number of peaks in the mass range of $800-3000 \mathrm{~m} / \mathrm{z}$. The combination of ion mobility and MALDI-TOF imaging, in the example shown in Figure 4, allows the separation of isobaric peptides and lipids. As such, it combines molecular imaging with direct identification of many different peptides in the tissue.

Ion-mobility mass spectrometry generates extra analytical information used for protein conformer and nominally isobaric structural identification. The added value of the IM-MS is the power to selectively isolate and identify lipids, peptides, and now proteins in a complex mixture. 
(a)
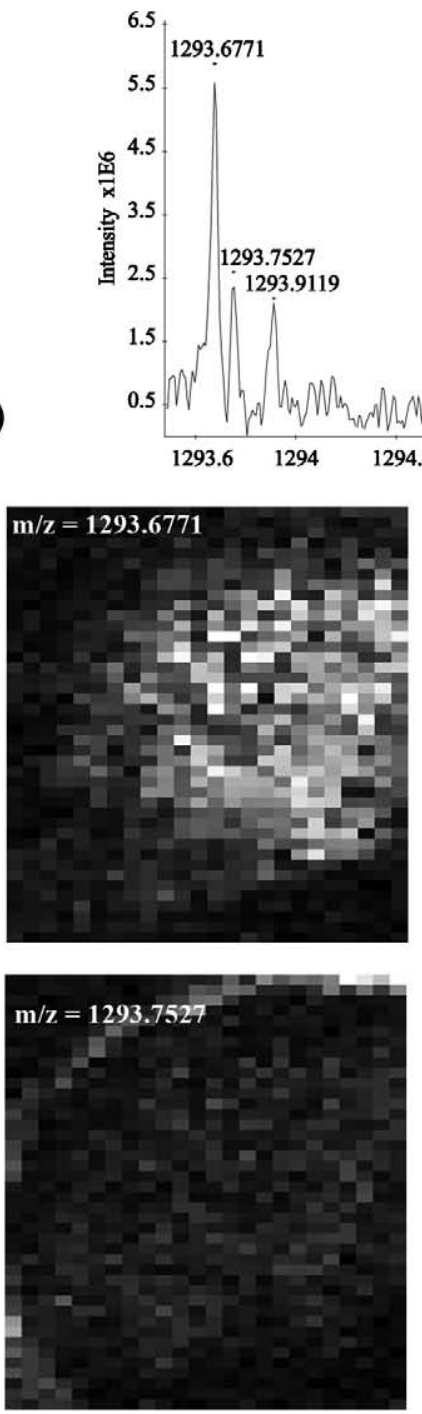

(b)

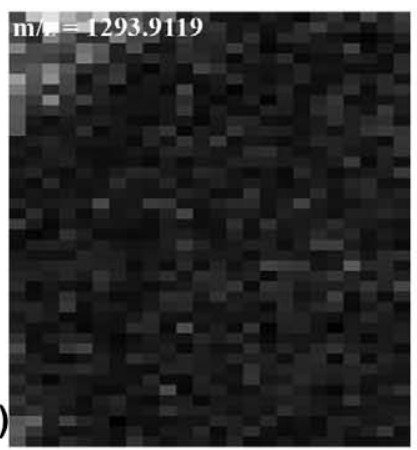

1294.6813
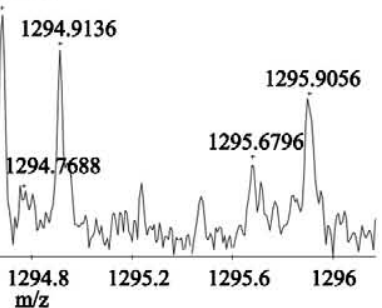

$$
\begin{array}{l|l}
11.5 & 1293.6809 \\
10.5 &
\end{array}
$$$$
9.5
$$

8.5
7.5

र 6.5

急 6.5

兽 4.5

4.5

2.5

1.5
0.5

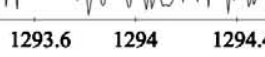

1294.6876

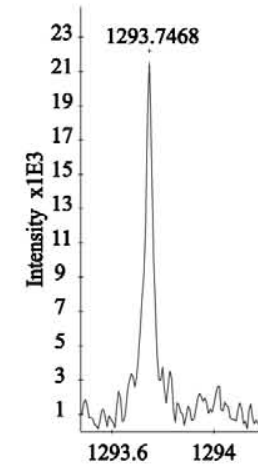

$\mathrm{m} / \mathbf{z}$

(d) 4
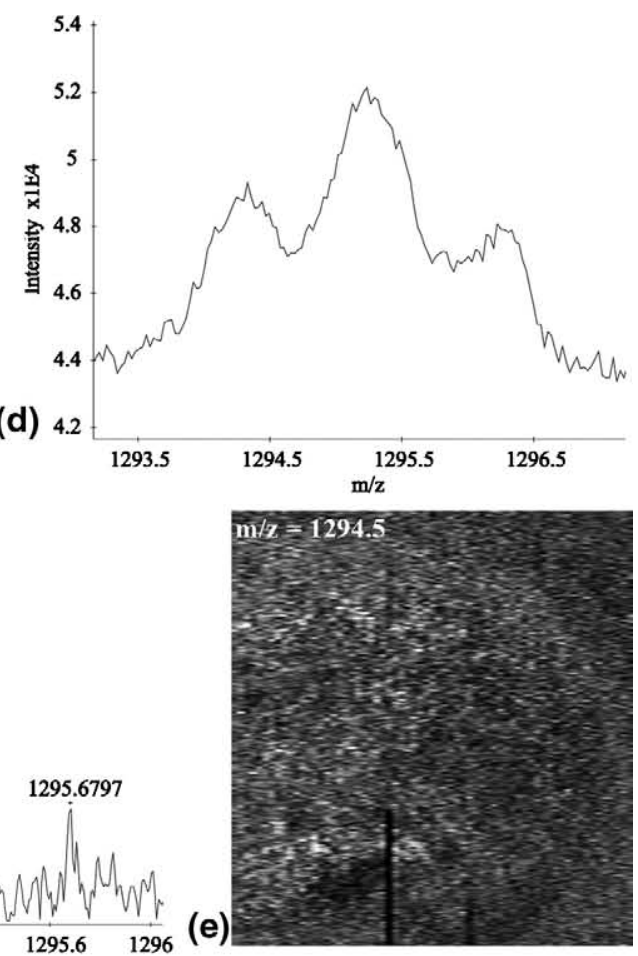

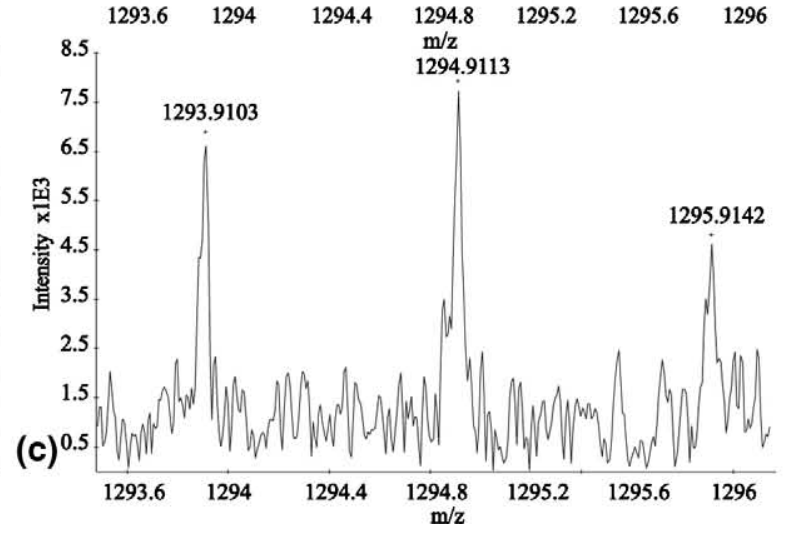

Figure 3. (a) Selected region of the FTICR summed spectrum of half of a rat brain tissue section. (b) FTICR selected ion images of the first isotope of three different compounds. (c) Region of interest analysis reveals the spectrum of each individual component. (d) Selected region of the summed spectrum of half of the rat brain tissue section. (e) The TOF selected ion image of $m / z=1294$. Because of a technical problem, a part of a line scan of TOF experiments is not present in the selected ion images. 


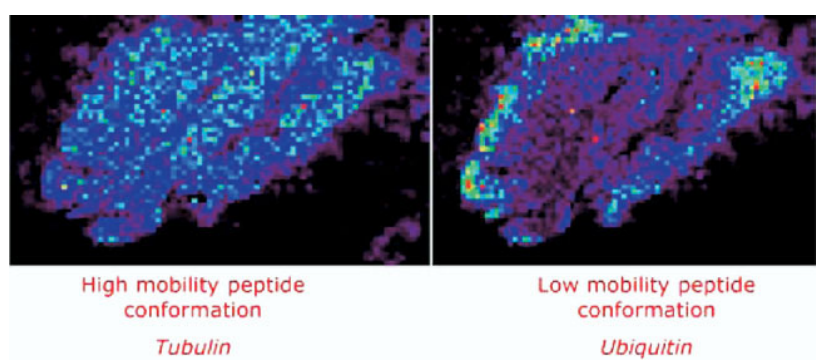

Figure 4. On-tissue identification and repartition of two nominally isobaric tryptic peptides at $\mathrm{m} / \mathrm{z} 1039$ coming from a enzymatic digestion of a FFPE rat brain analyzed with MALDI ion mobility MS imaging.

\section{Sample Preparation for Imaging Mass Spectrometry}

\section{General Sample Preparation and Matrix Deposition}

The quality of the sample surface is the first concern when performing imaging MS. The surface needs to be prepared ideally such that the original spatial distribution of molecules of interest is not compromised. In addition, imaging MS critically depends on efficient desorption and ionization of the surface molecules. To increase the ion yield from biological surfaces or access specific classes of molecules, surface modifications are often required. It is important to find tissue treatments that ensure reproducible and high-quality label-free molecular images. At this point in time, sample preparation is one of the most critical steps to obtain high MS image quality directly from tissue sections. A number of factors are identified that contribute to this goal: sample collection and storage, sample pretreatment, surface coverage with matrix solutions, and methods for matrix application.

Proper treatment of tissues begins with the surgical removal of tissue in a manner that avoids possible delocalization and degradation of the peptides and proteins. It is beneficial to inactivate enzymes through denaturation. This prevents the nonspecific degradation of intact proteins. Freshly harvested/denatured and frozen samples should subsequently be cryo-sectioned in thin slices in a contamination-free environment before the experiments are performed.

A sound washing protocol with various organic solvents applied to the tissue sections, before the experiments, is the next important step [24]. This procedure improves the quality of the signal coming from the tissue sections. The composition of the washing solvent mixture influences the content of chemical information obtained from the tissue surface, and each combination of analysis and tissue washing protocols should be carefully chosen and optimized for imaging MS experiments. The choice of the matrix solution is the next essential element of sample pretreatment in imaging MALDI (and occasionally ME-SIMS) experiments. The matrix solution should form a homogeneous layer of matrix-analyte crystals smaller than the tissue features that can be observed, given the spatial resolution of the imaging mass spectrometer used. As for the matrix choice, along with conventional matrices such as SA, CHCA, and DHB, also solid/liquid ionic matrices [25] are demonstrated to be good matrices for imaging MS.

A proper matrix application technique is another critical element of pretreatment. Aspects such as homogeneity of the applied matrix layer and reproducibility of matrix application to droplet size, speed, surface wetting, and cost critically determine the matrix deposition method chosen [26]. A high spatial resolution approach requires the use of small crystals, typically smaller than the pixel size in the final image. Unfortunately, this often compromises sensitivity because the extraction capability of smaller droplets is poor. Environmental factors such as temperature and humidity are also important parameters that need to be controlled during matrix deposition. The deposition of matrix on tissue that is desiccated while the sample is brought from $-80^{\circ} \mathrm{C}$ to room temperature for matrix deposition will result in limited sensitivity. This is a result from the migration of surface components to the back of the tissue section when the sample becomes rehydrated upon first interaction with the matrix solution. When all these sample preparation requirements are properly taken into consideration, imaging MS can be reproducible and routine. Only in that case is it fulfilling the potential that researchers hope for and can be considered a powerful new tool for biomedical proteomics.

\section{On-Tissue Digestion and Surface Chemistry}

In imaging mass spectrometry the sensitivity for intact proteins is generally low because of the complexity of the surface. This results in the generation of images of only abundant intact proteins such as for instance histones, myelin, and tubulin in brain tissue. Moreover, identification of these abundant proteins is complicated by the post-translational modifications, splice variants, and different adducts that can be observed. A different approach is needed to at least partly overcome this problem. A powerful complementary technique to direct protein analysis is on-tissue enzymatic digestion [5, 26-28]. Protein digestion typically yields peptides between 400 and $3500 \mathrm{Da}$, a range in which instrumental sensitivity is higher than that for intact proteins. However, the mass spectrum is more complicated in the low-mass region. In addition, enzymatic digestion enables efficient MS/MS on small singly charged peptides in ToF-based instruments. Current approaches for identification of digested proteins or peptides from tissue involve extraction and separate MS/MS measurements, sometimes followed by comparison with on-tissue peptide mass fingerprinting (PMF) [26]. Tissue chemical complexity prohibits efficient PMF. Further, the massresolving power and mass accuracy of ToF instruments are usually insufficient for exact mass detection of all measured intact proteins, although FT-ICR MS and 
ion-mobility-based methods can be used as discussed earlier [15]. MS/MS has scarcely been performed directly on tissue-typically on the most abundant peptides [27] or to distinguish between isobaric compounds rather than absolute identification [29, 30]. However, the chemical complexity on tissue may cause multiple peptides at each nominal mass. As a consequence, except for higher abundant peptides, identification by MS/MS may prove difficult as a result of the fragments from different peptides. To overcome this issue chemical derivatization of the peptides combined with a post-ionization strategy could enhance the identification capabilities for these types of studies [31]. The enhanced sequencing capabilities using MS/MS offered by the application of tri-methoxyphenylphosphonium (TMPP) derivatives [32] on digested tissue surfaces is a potential alternative method to overcome the identification sensitivity issues. From an instrumental perspective ultrahigh resolution FT-ICR MS imaging or gasphase ion mobility separation before MS/MS can assist in tryptic peptide identification.

These on-tissue digestion and derivatization protocols involve liquid handling and the deposition of large droplets (typically, $100 \mu \mathrm{m}$ in diameter) using robotic deposition methods. Once again, great care has to be taken to control temperature, surface salt content, and humidity during the digestion process. All spatial information within the droplets is lost during the digestion process. On-tissue digestion is therefore mostly suited for use in combination with lower-resolution techniques. New digestion protocols are continuously being developed that can also be used for MALDI imaging MS [33].

\section{Challenge the Picture: Ion Suppression}

Does a measured image quantitatively reflect the variation in concentration of a specific molecule? In other words: what is the value of a mass-resolved image? This is a question that is very difficult to answer as a result of the ion-suppression effect. Ion suppression is a term that refers to the fact that surface ionization processes do not result in identical ionization efficiencies for each chemical species on the surface. The ionization efficiency and thus the analyte intensities depend on the local chemical environment [34]. This makes it nearly impossible to generate quantitative mass spectrometric images without evaluating the ion-suppression effects for the compounds of interest on the tissue under study. This applies both for SIMS [35] and MALDI-MS molecular imaging. The best example is given by the comparison between a gradient of matrix-to-analyte ratio on a surface and in the resulting mass spectra [36]. Specific ionization yields in MALDI are hard to predict because they are the result of numerous equilibria $[37,38]$ that are influenced by a high degree of chemical complexity of biological tissues.

The ion-suppression effect is a major obstacle to quantification in many analytical disciplines. The inter-

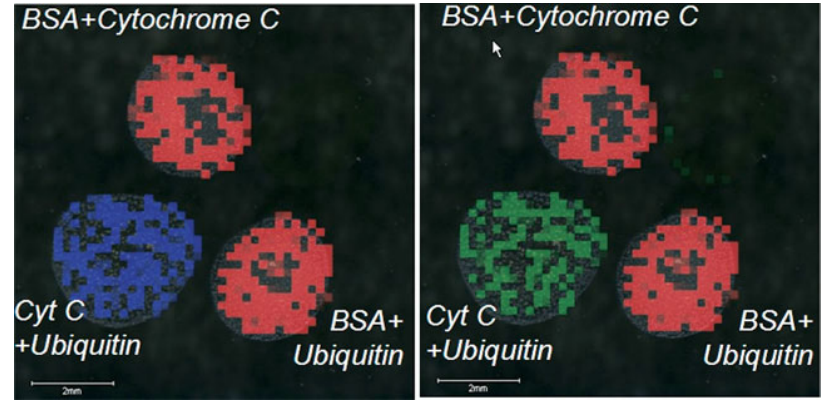

Figure 5. MS image of peptides identified by MS/MS from digests of BSA (red), ubiquitin (green) and cytochrome C (blue). BSA completely suppresses the signal from the 2 other protein digests.

pretation of mass spectrometric images is intrinsically quantitative, which confers an added importance to this phenomenon. The MS image of a biological surface appears to display the differential relative concentration between analytes, which is precisely the result we want to observe. Here we run into a chicken-and-egg problem. We want to observe the spatial distribution of molecules, yet the spatial distribution of these molecules can influence the outcome of the experiment. Figure 5 illustrates the effect of a different chemical environment on the resulting mass spectra: three aqueous protein digest solutions (bovine serum albumin [BSA], cytochrome C, and ubiquitine) are spotted two by two in identical concentrations with a saturated matrix solution of HCCA in water:acetonitrile (30:70 and TFA $0.1 \%$ ). Images are acquired by MALDI-ToF imaging with an ULTRAFLEX-III (Bruker Daltonik $\mathrm{GmbH}$, Bremen, Germany) using the associated Bruker FlexImaging software. Specific images are produced by selection of peaks specific for each of the protein digests. Although cytochrome $\mathrm{C}$ and ubiquitine appear simultaneously, they do not show up where BSA was spotted, illustrating a strong suppression of BSA digest on both of the other peaks.

Considering the potential impact on the interpretation of a mass image, sample preparation protocols need to be adapted to limit the effect of ion suppression. To counteract the ion-suppression effect of salts, washing the tissue with polar solvent is very commonly performed (see sample preparation). Lipid removal, used for peptides and protein imaging, is done by washing tissue with apolar solvents like chloroform [24]. In some cases it is possible to foresee the potential importance of the ion-suppression effect on a tissue of interest by homogeneously covering a test tissue section with known standard compound with a chemical structure similar to that of the analytes under study. A wide range of chemical environments are expected from different cell types on a single tissue section (e.g., stroma versus epithelial cells or gray versus white matter in brain). In some cases it is observed that there is no important ionization difference if the cell types are sufficiently similar and the main difference remains 
between tissue and, for instance, the glass slide outside tissue [26]. This implies that in spite of ion-suppression effects for selected compounds and selected tissues it is possible to perform relative quantitation.

\section{Summary}

Clearly, great care has to be taken when interpreting mass spectral images. The mere display of single mass images revealing an anatomical feature on tissue is slowly making way for interpreted images of identified endogenous biomolecules or pharmaceutical compounds. Imaging mass spectrometry has developed to a technique that can solve actual problems in biomedical research. Yet it has its limitations. Instrumentation developments for imaging MS are continuously removing technical barriers and limitations and result in an increase of its analytical value. Each implementation of imaging MS can visualize a small subset of all molecules present on the surface under study. Each implementation offers different sensitivities, spatial resolution, and speed of analysis. All MS imaging implementations need to be combined with other molecular imaging techniques and standard analytical protocols applied to tissue homogenates to provide positive identification of the molecules associated with a spatial structure. If researchers who move into this field take this and the effects of sample treatment/ handling into account then the technique is past the hype stage and is fulfilling its full potential. In the future we will see multiple new applications developing in the field of nanomedicine, pharmacokinetics, proteome imaging, and many others. It will take at least another 5 years to firmly state that the applications of imaging mass spectrometry have fulfilled the hope of the researchers in these fields. There is still a lot of work to be done-when you look at it critically!

\section{Acknowledgments}

This work is part of the research program of the "Stichting voor Fundamenteel Onderzoek der Materie (FOM)," which is financially supported by the Nederlandse organisatie voor Wetenschappelijk Onderzoek (NWO). The authors also acknowledge funding and collaborations through the Biomarker Discovery research program of the Netherlands Proteomics Center (NPC), COMPUTIS and MEDITRANS, both projects of the sixth European Framework Program for Research and Technological Development.

\section{References}

1. McDonnell, L. A.; Heeren, R. M. A. Imaging Mass Spectrometry. Mass Spectrom. Rev. 2007, 26, 606-643.

2. Caprioli, R. M.; Farmer, T. B.; Gile, J. Molecular Imaging of Biological Samples: Localization of Peptides and Proteins Using MALDI-TOF MS. Anal. Chem. 1997, 69, 4751-4760.

3. Stoeckli, M.; Chaurand, P.; Caprioli, R. M. Applications of MALDI MS Imaging to Biological Samples. J. Am. Soc. Mass Spectrom. 1999, 10, $1255-1260$.

4. Reyzer, M. L.; Hsieh, Y.; Ng, K.; Korfmacher, W. A.; Caprioli, R. M. Direct Analysis of Drug Candidates in Tissue by Matrix-Assisted Laser Desorption/Ionization Mass Spectrometry. J. Mass Spectrom. 2003, 38, 1081-1092.
5. Rohner, T. C.; Staab, D.; Stoeckli, M. MALDI Mass Spectrometric Imaging of Biological Tissue Sections. Mech. Ageing Dev. 2005, 126, 177-185.

6. Altelaar, A. F. M.; Luxembourg, S. L.; McDonnell, L. A.; Piersma, S. R.; Heeren, R. M. A. Imaging Mass Spectrometry at Cellular Length Scales. Nat. Protoc. 2007, 2, 1185-1196.

7. Heeren, R.M.A.; McDonnell, L. A.; Amstalden, E.; Luxembourg, S. L. Altelaar, A. F. M.; Piersma, S. R. Why Don't Biologists Use SIMS. A Critical Evaluation of Imaging MS. Appl. Surf. Sci. 2005, 252, 68276835.

8. Karas, M.; Bachmann, D.; Bahr, U.; Hillenkamp, F. Matrix-Assisted Ultraviolet Laser Desorption of Non-volatile Compounds. Int. J. Mass Spectrom. Ion Process. 1987, 78, 53-68.

9. Stoeckli, M.; Staab, D.; Schweitzer, A. Compound and Metabolite Distribution Measured by MALDI Mass Spectrometric Imaging in Whole-Body Tissue Sections. Int. J. Mass Spectrom. 2007, 260, 195-202.

10. Spengler, B.; Hubert, M. Scanning Microprobe Matrix-Assisted Laser Desorption Ionization (SMALDI) Mass Spectrometry: Instrumentation for Sub-micrometer Resolved LDI and MALDI Surface Analysis. J. Am. Soc. Mass Spectrom. 2002, 13, 735-748.

11. Jurchen, J. C.; Rubakhin, S. S.; Sweedler, J. V. MALDI-MS Imaging of Features Smaller Than the Size of the Laser Beam. J. Am. Soc. Mass Spectrom. 2005, 16, 1654-1659.

12. Luxembourg, S. L.; Mize, T. H.; McDonnell, L. A.; Heeren, R. M. A. High-Spatial Resolution Mass Spectrometric Imaging of Peptide and Protein Distributions on a Surface. Anal. Chem. 2004, 76, 5339-5344.

13. Luxembourg, S. L.; McDonnell, L. A.; Mize, T. H.; Heeren, R. M. A. Infrared Mass Spectrometric Imaging below the Diffraction Limit. J. Proteome Res. 2005, 4, 671-673.

14. Marshall, A. G.; Hendrickson, C. L.; Jackson, G. S. Fourier Transformation Ion Cyclotron Resonance Mass Spectrometry: A Primer. Mass Spectrom. Rev. 1998, 17, 1-35.

15. Taban, I. M.; Altelaar, A. F. M.; van der Burgt, Y. E. M.; McDonnell L. A.; Heeren, R. M. A.; Fuchser, J.; Baykut, G. Imaging of Peptides in the Rat Brain Using MALDI-FTICR Mass Spectrometry. J. Am. Soc. Mass Spectrom. 2007, 18, 145-151.

16. Kutz, K. K.; Schmidt, J. J.; Li, L. J. In Situ Tissue Analysis of Neuropeptides by MALDI FTMS In-Cell Accumulation. Anal. Chem. 2004, 76, 5630-5640.

17. Stemmler, E. A.; Cashman, C. R.; Messinger, D. I.; Gardner, N. P. Dickinson, P. S.; Christie, A. E. High-Mass-Resolution Direct-Tissue MALDI-FTMS Reveals Broad Conservation of Three Neuropeptides (APSGFLGMRamide, GYRKPPFNGSIFamide and pQDLDHVFLRFamide) across Members of Seven Decapod Crustaean Infraorders. Peptides 2007, $28,2104-2115$.

18. Stemmler, E. A.; Hsu, Y. W.; Cashman, C. R.; Messinger, D. R.; de la Iglesia, H. O.; Dickinson, P. S.; Christie, A. E. Direct Tissue MALDIFTMS Profiling of Individual Cancer Productus Sinus Glands Reveals That One of Three Distinct Combinations of Crustacean Hyperglycemic Hormone Precursor-Related Peptide (CPRP) Isoforms Are Present in Individual Crabs. Gen. Comp. Endrocrinol. 2007, 154, 184-192.

19. Cornett, D. S.; Frappier, S. L.; Caprioli, R. M. MALDI-FTICR Imaging Mass Spectrometry of Drugs and Metabolites in Tissue. Anal. Chem. 2008, 80, 5648-5653.

20. Kingshott, P. for the editorial staff at Kratos Analytical. Surface-MALDI Investigation of Protein Adsorption to Contact Lens Surfaces. Kratos Analytical Application Note. Available at http://www.kratos.com/ Mapps/NewApps/Apps23.html.

21. Clemmer, D. E.; Jarrold, M. F. Ion Mobility Measurements and Their Applications to Clusters and Biomolecules. J. Mass Spectrom. 1997, 32, 577-592.

22. Woods, A. S.; Ugarov, M.; Egan, T.; Koomen, J.; Gillig, K. J.; Fuhrer, K.; Gonin, M.; Schultz, J. A. Lipid/Peptide/Nucleotide Separation with MALDI-Ion Mobility-TOF MS. Anal. Chem. 2004, 76, 2187-2195.

23. Tempez, A.; Ugarov, M.; Egan, T.; Schultz, J. A.; Novikov, A.; Della-Negra, S.; Lebeyec, Y.; Pautrat, M.; Caroff, M.; Smentkowski, V. S.; Wang, H. Y.; Jackson, S. N.; Woods, A. S. Matrix Implanted Laser Desorption Ionization (MILDI) Combined with Ion MobilityMass Spectrometry for Bio-Surface Analysis. J. Proteome Res. 2005, 4 , 540-545.

24. Lemaire, R.; Wisztorski, A.; Desmons, A.; Tabet, J. C.; Day, R.; Salzet, M.; Fournier, I. MALDI-MS Direct Tissue Analysis of Proteins: Improving Signal Sensitivity Using Organic Treatments. Anal. Chem. 2006, 78, 7145-7153.

25. Lemaire, R.; Tabet, J. C.; Ducoroy, P.; Hendra, J. B.; Salzet, M.; Fournier I. Solid Ionic Matrixes for Direct Tissue Analysis and MALDI Imaging. Anal. Chem. 2005, 78, 809-819.

26. MacAleese, L.; Stauber, J.; Heeren, R. M. A. Perspectives for Imaging Mass Spectrometry in the Proteomics Landscape. Proteomics Rev. 2009, 9, 819-834.

27. Groseclose, M. R.; Andersson, M.; Hardesty, W. M.; Caprioli, R. M. Identification of Proteins Directly from Tissue: In Situ Tryptic Digestions Coupled with Imaging Mass Spectrometry. J. Mass Spectrom. 2007, $42,254-262$.

28. Lemaire, R.; Desmons, A.; Tabet, J. C.; Day, R.; Salzet, M.; Fournier, I. Direct Analysis and MALDI Imaging of Formalin-Fixed, ParaffinEmbedded Tissue Sections. J. Proteome Res. 2007, 6, 1295-1305.

29. Garrett, T. J.; Prieto-Conaway, M. C.; Kovtoun, V.; Bui, H.; Izgarian, N. Stafford, G.; Yost, R. A. Imaging of Small Molecules in Tissue Sections 
with a New Intermediate-Pressure MALDI Linear Ion Trap Mass Spectrometer. Int. J. Mass Spectrom. 2007, 260, 166-176.

30. Shimma, S.; Sugiura, Y.; Hayasaka, T.; Zaima, N.; Matsumoto, M.; Setou, M. Mass Imaging and Identification of Biomolecules with MALDI-OIT-TOF-Based System. Anal. Chem. 2008, 80, 878-885.

31. Edirisinghe, P. D.; Moore, J. F.; Skinner-Nemec, K. A.; Lindberg, C.; Giometti, C. S.; Veryovkin, I. V.; Hunt, J. E.; Pellin, M. J.; Hanley, L. Detection of In Situ Derivatized Peptides in Microbial Biofilms by Laser Desorption $7.87 \mathrm{eV}$ Postionizaton Mass Spectrometry. Anal. Chem. 2007, 79, 508-514.

32. Kuyama, H.; Sonomura, K.; Shima, K.; Nishimura, O.; Tsunasawa, S. An Improved Method for De Novo Sequencing of Arginine-Containing, $\mathrm{N}^{\alpha}$-tris(2,4,6-Trimethoxyphenyl)phosphonium-Acetylated Peptides. Rapid Commun. Mass Spectrom. 2008, 22, 2063-2072.

33. Taouatas, N.; Drugan, M. M.; Heck, A. J. R.; Mohammed, S. Straightforward Ladder Sequencing of Peptides Using a Lys-N Metalloendopeptidase. Nat. Methods 2008, 5, 405-407.
34. Luxembourg, S. L.; McDonnell, L. A.; Duursma, M. C.; Guo, X.; Heeren, R. M. A. Effect of Local Matrix Crystal Variations in Matrix-Assisted Ionization Techniques for Mass Spectrometry. Anal. Chem. 2003, 75, 2333-2341.

35. Jones, E. A.; Lockyer, N. P.; Kordys, J.; Vickerman, J. C. Suppression and Enhancement of Secondary Ion Formation Due to the Chemical Environment in Static-Secondary Ion Mass Spectrometry. J. Am. Soc. Mass Spectrom. 2007, 18, 1559-1567.

36. Knochenmuss, R.; Karbach, V.; Wiesli, U.; Breuker, K.; Zenobi, R. The Matrix Suppression Effect in Matrix-Assisted Laser Desorption/ Ionization: Application to Negative Ions and Further Characteristics. Rapid Commun. Mass Spectrom. 1998, 12, 529-534.

37. Knochenmuss, R.; Zenobi, R MALDI Ionization: The Role of In-Plume Processes. Chem. Rev. 2003, 103, 441-452.

38. Lehmann, E.; Knochenmuss, R.; Zenobi, R. Ionization Mechanisms in Matrix-Assisted Laser Desorption Ionization Mass Spectrometry: Contribution of Pre-formed Ions. Rapid Commun. Mass Spectrom. 1997, 11 1483-1492. 